\section{SARS-Cov-2 and drug shortages - Trends and lessons from a Caribbean perspective}

Satish Jankie ${ }^{1}$, Shalini Pooransingh ${ }^{2}$, Arlene Villaroel Stuart $^{1}$, Lexley Maureen Pinto Pereira ${ }^{2}$

${ }^{1}$ School of Pharmacy, ${ }^{2}$ Department of Para-clinical

Sciences, Faculty of Medical Sciences, The University of the West Indies, St, Augustine, Trinidad and Tobago.

\section{Corresponding author:}

Dr. Satish Jankie, PhD

School of Pharmacy

Faculty of Medical Sciences

St. Augustine

Trinidad and Tobago

Telephone/Fax: 1-868-662-1472

Email: satish.jankiet@sta.uwi.edu

DOI: $10.48107 / C M J .2020 .11 .002$

Copyright:

(c) (i)

This is an open-access article under the terms of the Creative Commons Attribution License which permits use, distribution, and reproduction in any medium, provided the original work is properly cited.

\section{INTRODUCTION}

Coronavirus SARS-CoV-2 first reported in Wuhan, China in December 2019 has rapidly infected millions, claimed the lives of a large global population, crippled economies, created a new 'normal' and continues to flog the health care system. The pandemic seems to have affected supply and demand of pharmaceutical entities as an early concern was that medications for patients on ventilators would soon run out. Critical points for management of the pandemic were highlighted in China and even with adequate ICU beds and frontline workers, shortage of emergency medications remained a major concern (1). As the surge began, sedatives and painkillers were soon in short supply at hospital pharmacies and clinical pharmacists began to spend more time on drug acquisition and conservation than on patient care (2). While drug shortages are not uncommon, the titanic surge of SARS-CoV-2 has led to the use of alternative drugs in place of first line analgesics, neuromuscular blockers, vasopressors and antimicrobials in the United States (3). Europe is experiencing scarcities of critical drugs needed to manage patients with COVID-19 such as sedatives, analgesics, bronchodilators, antimicrobials and antimalarial agents (4). The American Society of HealthSystem Pharmacists (ASHP) has been conducting weekly reports on drug availability and has proposed methods for therapeutic assessment, drug shortage impact analysis, and alternate available options (3). Clinical pharmacists have been actively identifying drugs in short supply, considering alternatives and assessing the risks when using these agents. They have also been monitoring drug use and reducing drug wastage, which is of paramount importance when there are drug shortages.

With increasing patient numbers overwhelming the hospitals, availability of drugs decreased and the number of prescriptions being filled for sedatives, muscle relaxants, anesthetic agents for the pain and distress in mechanically ventilated patients dropped by $4-7 \%$ in just one week and analgesic prescriptions stayed at $69 \%$ (5). The United States Drug Enforcement Agency (DEA) promptly approved a $15 \%$ increase in production quotas for controlled substances like fentanyl, morphine, hydromorphone, codeine, ephedrine, and pseudoephedrine, and in parallel will permit increased 
imports of ketamine, diazepam, midazolam, lorazepam, and phenobarbital (6).

\section{DISCUSSION}

Drug shortages- why do they occur?

The COVID-19 pandemic has worsened some shortages and created others. Roughly half of all drugs in shortage are injectable, including analgesics, sedatives and paralytics needed to intubate critically ill patients (3). Shortages in drug supply are generally consequent to issues with quality control, such as when a product may be recalled or destroyed. Drugs with low frequency use or demand are manufactured in limited amounts, and shortages are precipitated in an acute high demand scenario. The pandemic has triggered sudden global demands for certain pharmaceuticals, placing a high burden on manufacturers- some of whom are adversely affected by the pandemic themselves and forced to shut down operations, due to the inability to source raw materials and active pharmaceutical ingredients (APIs).

There is also the artificial shortage from claims of efficacy of agents purported to treat COVID-19.

Hydroxychloroquine has dominated sales beyond its true indications despite robust evidence of its efficacy in COVID-19 (7). Like a grocery item, fueled by anecdotal media reports and touted as a game changer, it underwent panic buying and stockpiling for possible treatment and even prophylaxis (8). As of June $8^{\text {th }}$ 2020, if the planned 212 clinical trials worldwide go ahead, patients, who rely on hydroxychloroquine for its licensed indications of rheumatoid arthritis and systemic lupus erythematosus (SLE) will face an escalated shortage. The increased demand for enoxaparin to treat COVID-19 associated coagulation disorders in the United Kingdom, amidst acute scarcity and quality issues saw forced imports from Italy (9). The National Health Service (NHS) subsequently banned the export of 82 drugs out of the UK to preserve supplies for their patients (10). India also restricted export of 26 drugs and drug ingredients, amid disruption of services at its manufacturing plants, prioritizing supply for its own people, provoking drug shortages worldwide, though India later opened her doors to exports (11).

Recognizing the projected impact of COVID-19 on drug shortages the NHS, England noting that community pharmacies are running out of supplies urged doctors not to overprescribe or over-order during this time, to avoid further pressures on the supply chain (12). Many of these reports address the acute shortage, but the reduced availability of APIs could mean chronic disease medications may be in the 'out of stock' list soon.

\section{Active pharmaceutical ingredients}

When China was recognized as the epicenter of the pandemic, there were concerns about the availability of raw materials and APIs for drug manufacture. China's supply of APIs has more than doubled in the last decade to $13 \%$ of the pharmaceutical market in the USA. The Indian pharmaceutical industry, the world's third largest drug manufacturer of medications at affordable prices, supplies medicines to a vast portion of the global population, and supplies more than 250 US FDA and UK Medicine and Healthcare Products Regulatory Agency (MHRA) approved plants (12). The majority of APIs for generic drug manufacturing globally are sourced from India, which also supplies approximately 30 percent of the generic APIs in the USA; COVID-19 has highlighted global dependence on these two pharmaceutical titans putting severe strain on the worlds Pharmaceutical Industry (13).

Trinidad and Tobago has experienced the situation of anxious patients hoarding medications, anticipating the fury of COVID-19 will threaten drug supplies particularly for chronic non-communicable diseases. An overnight $300 \%$ hike in drug prices for some agents, could not be controlled by the local Pharmacy Board which passed the blame to the wholesalers (14). Trinidad and Tobago's Minister of Health made an impassioned public appeal in one of the daily press conferences of the Ministry of Health, urging retailers to desist from spiraling prices and even suggested patients hoarding hydroxychloroquine should return supplies to the retail pharmacy so that patients with autoimmune disorders could avail their treatment without financial liability.

\section{Challenges- counterfeit medications}

The dearth of much needed pharmaceuticals has opened doors for the entry of counterfeit medications. Patients generally do not suspect counterfeit or substandard 
medicines when doing online shopping. To highlight the significance and dangers of fake medicines, officials from 90 countries during 3 - 10 March 2020, inspected over 326,000 packages and seized more than 48,000 , with a total worth of 4 billion US dollars (15). A total of 4.4 million units of illicit pharmaceuticals were seized worldwide. The most common items detected were erectile dysfunction pills, anti-cancer medication, hypnotic and sedative agents, anabolic steroids, analgesics/ painkillers, nervous system and dermatological agents and vitamins. These bogus agents may also be found on pharmacy shelves in some countries where it is illegally imported, putting patients at high risk for adverse events. Thus, the unsuspecting consumer would experience worsening of their chronic disease, additionally burdening the health care system.

\section{Can drug shortages be mitigated?}

COVID-19 is likely to persist for the foreseeable future, mandating the critical management of scarce resources to ensure optimal patient care and to protect public health. There should be equitable access to drugs so that those who require drugs for specific conditions are able to obtain them, rather than bulk-buying by those with the means to do so. Allowing importation of generic drugs with proven quality assurance studies from approved manufacturers can prevent shortages and even control prices. Prescribing proven generics should be encouraged and pharmacists can educate patients on their costefficacy, if the prescription does not forbid switching the brand. Pharmaceutical manufacturers can be requested to repurpose their production facilities to manufacture generic drugs that are facing shortages, similar to General Motors shifting to ventilators in the best interest of the nation's health. Measures should be implemented to produce generic drugs with small profit margins as these are unattractive to pharmaceutical companies despite their critical need. Responsible reporting of the results of drug trials and of potential candidates for treating COVID-19 can prevent panic bulk buying which causes societal health inequity from artificial drug shortages and price increases, which will have negative implications for medications unrelated to the treatment of COVID-19.

\section{CONCLUSION}

The Ministry of Health in conjunction with the Pharmacy Board can develop policies in the short term to maximize available pharmaceuticals with minimal wastage in treating COVID-19 patients. Public education is imperative for the unsuspecting consumer and the community to be made aware of any impending drug shortages with recommended changes in therapy to prevent the unknowing purchase of false products. A major issue for global consideration is the dependence on just a few nations for the bulk of the world's supply of APIs. Serious consideration by relevant stakeholders can encourage and support more centres for worldwide pharmaceutical manufacturing, to ensure a reliable supply of medications in the event that the current plants are unable to operate. Measures to ensure that patients can access good quality, low priced pharmaceuticals to contain chronic diseases and avoid overwhelming the health care system during or post- COVID-19 scene, would be a welcome initiative by health authorities and care givers.

\section{References}

1. Li, L., Gong, S. \& Yan, J. COVID-19 in China: ten critical issues for intensive care medicine. Crit Care 24, 124 (2020). DOI: https://doi.org/10.1186/s13054 $-020-02848-z$

2. Davies M, Stockton B, Furneaux R. Drug shortages put worst-hit Covid-19 patients at risk. The Bureau of Investigative Journalism. April 9, 2020. Accessed:

April 14 2020. Available: https:// www.thebureauinvestigates.com/stories/2020-04-09/ drug-shortages-put-worst-hit-covid-19-patients-at-risk

3. Fox ER, McLaughlin MM. ASHP guidelines on managing drug product shortages. Am J Health Syst Pharm. 2018;75(21):1742-50. DOI: 10.2146/ ajhp180441

4. Robinson J. Supplies of some COVID-19 medicines to run out within days, government warns. The Pharmaceutical Journal. April 20, 2020. Accessed April 20, 2020. Available : https:// www.pharmaceutical-journal.com/news-and-analysis/ news/supplies-of-some-covid-19-medicines-to-run-out -within-days-government-warns/20207909.article? 
firstPass $=$ false

5. Silverman E. As demand surges, half of prescriptions needed for Covid-19 patients on ventilators are going unfilled. Pharmalot. April 15, 2020. Accessed: April 20,2020. Available: https://www.statnews.com/ pharmalot/2020/04/15/covid19-coronavirusventilators-drug-shortages/

6. Choo EK, Rajkumar SV. Medication Shortages During the COVID-19 Crisis: What We Must Do. Mayo Clinic proceedings. January 1, 2020. Accessed: February 23, 2020. DOI: https://doi.org/10.1016/ j.mayocp.2020.04.001

7. Mehta B, Salmon J, Ibrahim S. Potential Shortages of Hydroxychloroquine for Patients with Lupus During the Coronavirus Disease 2019 Pandemic. JAMA Health Forum. 2020; 1(4): e200438. DOI: 10.1001/ jamahealthforum.2020.0438

8. Parkinson J, Gauthier-Villars D. Trump claim that malaria drugs treat Coronavirus sparks warnings, shortages. The Wall Street Journal. Updated March 23, 2020. Accessed March 30, 2020. Available: https://www.wsj.com/articles/trump-claim-thatmalaria-drugs-treat-coronavirus-sparks-warningsshortages-11584981897

9. Finch I. Italian batches of Clexane imported to cope with UK shortage. Community pharmacy news, analysis and CPD. January 24, 2020. Accessed: March 18, 2020. Available : https:// www.chemistanddruggist.co.uk/news/italian-clexaneimported-after-quality-issue-identified-uk-batch

10. The UK Government, List of medicines that cannot be parallel exported from the UK. Updated 20 March 2020. Accessed March 24' 2020. Available: https:// assets.publishing.service.gov.uk/government/ uploads/system/uploads/attachment_data/ file/874142

11. Blankenship K. India pharma manufacturing hub back up and running after COVID-19 lockdown: report. Fierce Pharma. April 27, 2020.Accessed April 28, 2020. Available: https://www.fiercepharma.com/ manufacturing/india-pharma-manufacturing-hub-back -up-and-running-after-covid-19-lockdown-report

12. Stewart K, Connelly D, Robinson J. Everything you should know about the coronavirus outbreak. The Pharmaceutical Journal. June 11, 2020. Accessed June 16, 2020. Available: https:// www.pharmaceutical-journal.com/news-and-analysis/ features/everything-you-should-know-about-thecoronavirus-outbreak/20207629.article

13. Chatterjee, P. Indian pharma threatened by COVID19 shutdowns in China. The Lancet.2020;395,10225, 675. DOI: 10.1016/S0140-6736(20)30459-1

14. Gioannetti A. Deyalsingh: Drug hoarders hurting genuine patients. Newsday. April 6, 2020. Accessed April 14, 2020. Available: https:// newsday.co.tt/2020/04/06/deyalsingh-drug-hoardershurting-genuine-patients/

15. INTERPOL. Global operation sees a rise in fake medical products related to COVID-19. Coronavirus outbreak sparks a new trend in counterfeit medical items. March 2020. Accessed: April 2, 2020. Available: https://www.interpol.int/News-and-Events/ News/2020/Global-operation-sees-a-rise-in-fakemedical-products-related-to-COVID-19 
